\title{
Introducción Precoz de Sustitutos de Lactancia Materna e Incidencia de Lactancia Materna Exclusiva al Mes de Vida
}

\author{
INGRID CARDOSO K. ${ }^{1}$, PAULINA TOSO M. ${ }^{1}$, VERÓNICA VALDÉS L. ${ }^{1}$, \\ JAIME CERDA L. ${ }^{2}$, VALENTINA MANRÍQUEZ T.*, GONZALO PAIVA C.* \\ 1. Departamento de Pediatría, Facultad de Medicina, Pontificia Universidad Católica de Chile. \\ 2. Departamento de Salud Pública, Facultad de Medicina, Pontificia Universidad Católica de Chile. \\ * Internos de Pediatría, Facultad de Medicina, Pontificia Universidad Católica de Chile.
}

\begin{abstract}
Breast Milk Substitutes at One Month of Life and Breastfeeding Suspension Risk

Healthy term newborn feeding choice at the Maternity Ward may determine exclusive breastfeeding (EB) duration. Objective: Determine prospectively if early introduction of breast milk substitutes (BMS) or other liquids in healthy term newborns is associated to a reduction of EB incidence at 1 month of age. Methods: Concurrent cohort study of 211 healthy term neonates born in Hospital Clínico Universidad Católica during October 2007-June 2007, 108 non-supplemented and 103 supplemented with milk formula or dextrose 5\% during the time they stayed at the Maternity Ward. Crude and adjusted risk of EB cessation between both groups was estimated at 1 month of age. Results: One month after birth, 40/100 (40.0\%) of supplemented newborns and 26/101 (25.7\%) non-supplemented newborns were not receiving exclusive breastfeeding (crude $R R=1.55$ (CI95\% 1.03-2.34)). Adjusted by maternal characteristics (age, education, breastfeeding experience, education in breastfeeding, EB time projection and paternal support) and newborn characteristics (type of delivery and birthweight), EB cessation risk was 55\% higher in supplemented neonates (adjusted RR $=1.55, \mathrm{Cl} 95 \% 1.01$ 2.35). Conclusion: Early supplementation in healthy term newborns with BMS is associated to a higher risk of EB cessation at 1 month of age.
\end{abstract}

(Key words: Breastfeeding, newborn, cessation).

Rev Chil Pediatr 2010; 81 (4): 326-332

\section{RESUMEN}

Introducción: La forma de alimentación de un recién nacido de término (RNT) durante su estadía en la Maternidad puede condicionar la duración de la lactancia materna exclusiva (LME). Objetivo: Determinar prospectivamente si la introducción precoz de SLM (sustitutos de lactancia materna) u otros líquidos a RNT sanos se asocia a una reducción de la incidencia de LME al mes de vida. Métodos: Estudio de cohorte concurrente.

Trabajo recibido el 02 de septiembre de 2008, devuelto para corregir el 22 de octubre de 2008, segunda versión el 17 de mayo de 2010, aceptado para publicación el 01 de junio de 2010.

Correspondencia a:

Dra. Paulina Toso M.

E-mail:ptoso@med.puc.cl 
Se conformó una cohorte de 211 RNT sanos cuyo parto fue atendido en la Maternidad del Hospital Clínico Universidad Católica en el período octubre 2006-junio 2007, 108 no-suplementados y 103 suplementados con fórmula láctea o suero glucosado al 5\% durante su estadía en la Maternidad. Se estimó el riesgo crudo de cesación de LME al mes de vida entre los grupos no-suplementado vs suplementado, y ajustado por diversas variables confundentes. Resultados: Transcurrido un mes de vida, 40/100 (40,0\%) recién nacidos suplementados y 26/101 $(25,7 \%)$ recién nacidos no-suplementados habían dejado de recibir LME, siendo el RR crudo $=1,55$ (IC95\% 1,03-2,34). Ajustado por características maternas (edad, educación, experiencia previa de lactancia, educación en lactancia, proyección LME y apoyo paterno) y del recién nacido (vía de parto y peso de nacimiento), el riesgo de cesación de LME al mes de vida fue 55\% mayor en los RNT suplementados vs. no-suplementados (RR ajustado = 1,54; IC95\% 1,01-2,35). Conclusión: La suplementación precoz de RNT sanos con SLM asocia a un mayor riesgo de cese de LME al mes de vida.

(Palabras clave: Lactancia materna, recién nacidos, cesación).

Rev Chil Pediatr 2010; 81 (4): 326-332

\section{Introducción}

La lactancia materna exclusiva (LME) es la forma óptima de alimentación del lactante durante los primeros seis meses de vida, debido a los múltiples beneficios que aporta al binomio madre-hijo ${ }^{1-6}$. Uno de los factores que aumenta la duración de la LME es su promoción activa por parte del personal de salud ${ }^{7}$; no obstante, no es infrecuente la indicación de sustitutos de leche materna (SLM) u otros líquidos (e.g. suero glucosado $5 \%$ ) a recién nacidos por el mismo personal durante de los primeros días de vida ${ }^{8-10}$.

Diversos estudios han investigado los efectos de la introducción precoz de SLM u otros líquidos sobre el éxito y duración de la LME, reportándose un efecto negativo ${ }^{8,11-16}$. Por su parte, en 2005 se realizó una revisión sistemática de 56 ensayos clínicos randomizados sobre el tema, cumpliendo solamente uno de ellos los criterios de inclusión. Los autores concluyeron que a la fecha existía considerable incerteza sobre los efectos derivados de la introducción precoz de SLM u otros líquidos en el éxito y duración de la $\mathrm{LME}^{17}$, siendo de especial interés la falta de estudios metodológicamente bien diseñados. Por consiguiente, el objetivo de la presente investigación es determinar prospectivamente si la introducción precoz de SLM u otros líquidos a recién nacidos de término sanos se asocia a una reducción de la incidencia de LME al mes de vida, y cuanti- ficar la magnitud del riesgo de diversos factores asociados a esta práctica.

\section{Pacientes y Métodos}

\section{Tipo de estudio y sujetos de investigación}

El presente estudio tiene un diseño de tipo cohorte concurrente, correspondiendo su universo a los binomios madre-hijo cuyo parto fue atendido en la Maternidad del Hospital Clínico Universidad Católica en el período octubre 2006-junio 2007. Fueron incluidos recién nacidos de término sanos ( $>37$ semanas), dados de alta desde la Maternidad del hospital (i.e. no requirieron hospitalización por complicaciones) y que no contaran con contraindicaciones médicas para recibir leche materna (e.g. infección por VIH). La inclusión de cada binomio madre-hijo contó con el consentimiento informado de la madre. En base a la variable de exposición (i.e. introducción de SLM u otros líquidos durante la estadía del recién nacido en la Maternidad) se conformaron dos cohortes por conveniencia; una de recién nacidos no-suplementados $(\mathrm{n}=108)$ y otra de recién nacidos suplementados $(\mathrm{n}=103)$, ya sea con fórmula láctea o suero glucosado al $5 \%$, administrados por mamadera e indicados por el equipo de salud tratante. Mediante la revisión de fichas clínicas se obtuvieron datos demográficos de cada binomio madre-hijo y se preguntó a las madres su nivel de escolaridad 
( $\leq 12$ años/> 13 años de estudios), experiencia previa de amamantar (sí/no), educación prenatal sobre lactancia materna (sí/no), tiempo que desea amamantar ( $\leq 6$ meses/7 o más meses), vía de parto (vaginal o fórceps/cesárea) y existencia de apoyo paterno (sí/no), todos ellos de reconocida influencia sobre la duración de la LME, de aquí en adelante agrupados bajo el rótulo de "covariables" ocurrencia del evento principal de interés (i.e. persistencia de LME al mes de vida) se determinó mediante la aplicación de un cuestionario telefónico a la madre, realizado por un sólo médico (ICK), ciego a la exposición alimentaria del recién nacido durante su estadía en la Maternidad.

\section{Análisis estadístico}

Las variables categóricas fueron descritas en términos de número y frecuencia porcentual, y las variables numéricas en términos de promedio y desviación estándar. La normalidad de las variables fue evaluada mediante la prueba de Shapiro-Wilk.

En primer lugar, se analizó en forma cruda la asociación entre la principal variable de exposición (suplementación) y cada una de las covariables en estudio. Dada la naturaleza transversal de dicha asociación, ésta fue expresada en términos de odds ratio e intervalo de confianza 95\%. En segundo lugar, se analizó la asociación entre la principal variable de exposición (suplementación) y el evento de interés (cese de LME al mes de vida). Dado el sentido prospectivo de dicha asociación, ésta fue expresada en términos de riesgo relativo e intervalo de confianza 95\%. Esta asociación fue ajustada por aquellas covariables consideradas como confundentes (definidas como aquellas covariables cuya asociación con la principal variable de exposición presentó un valor $\mathrm{p}<$ $0,05)$, siendo incluidas en un modelo de regresión log binomial.

Por último, para caracterizar de mejor forma las covariables asociadas a la principal variable de exposición se construyó un modelo de regresión logística (modalidad 'Enter'), en el cual incluyeron únicamente aquellas covariables asociadas en forma univariada a la exposición principal. Los cálculos fueron reali- zados mediante el programa estadístico SPSS 15.0, considerándose estadísticamente significativo todo valor $\mathrm{p}<0,05$.

\section{Resultados}

Transcurrido un mes de vida, se dispuso información de 100 recién nacidos suplementados y 101 no-suplementados, siendo la pérdida total equivalente a 10 recién nacidos $(4,7 \%)$ (figura 1). Cuarenta recién nacidos suplementados $(40,0 \%)$ habían dejado de recibir LME, por su parte, $26(25,7 \%)$ recién nacidos nosuplementados habían dejado de recibir LME, siendo el RR crudo = 1,55 (IC95\% 1,03-2,34). En otras palabras, los recién nacidos suplementados presentaron 55\% más riesgo de cese de LME al mes de vida en comparación a los no-suplementados.

La tabla 1 resume las principales características de los binomios madre-hijo suplementados vs no-suplementados. De los recién nacidos suplementados, $53,4 \%$ recibió sólo un sus-

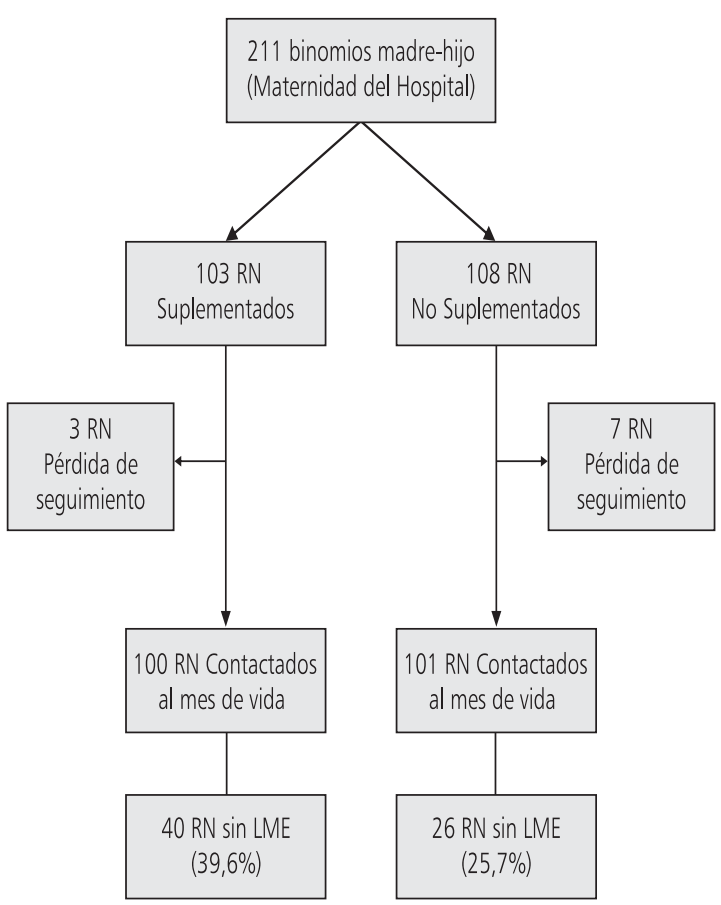

Figura 1. Seguimiento de la cohorte de binomios madrehijo. 
Tabla 1. Asociación univariada entre ocho covariables y la principal variable de exposición

\begin{tabular}{|c|c|c|c|c|}
\hline \multirow[t]{2}{*}{ Característica } & \multicolumn{2}{|c|}{$\begin{array}{l}\text { ¿Recibió suplemento de lactancia } \\
\text { materna en unidad de puerperio? }\end{array}$} & \multirow[t]{2}{*}{$\begin{array}{c}\text { OR } \\
\text { (IC 95\%) }\end{array}$} & \multirow[t]{2}{*}{ Valor $p$} \\
\hline & Sí & No & & \\
\hline \multicolumn{5}{|l|}{ Educ. materna } \\
\hline 13 o más años & 80 & 68 & 1,94 & 0,041 \\
\hline 0-12 años & 20 & 33 & $(0,97-3,88)$ & \\
\hline \multicolumn{5}{|l|}{ Experiencia previa LM } \\
\hline No & 55 & 49 & 1,30 & 0,358 \\
\hline Sí & 45 & 52 & $(0,72-2,35)$ & \\
\hline \multicolumn{5}{|l|}{ Educ. prenatal LM } \\
\hline No & 66 & 66 & 1,00 & 1,000 \\
\hline Sí & 34 & 34 & $(0,53-1,87)$ & \\
\hline \multicolumn{5}{|l|}{ Vía de parto } \\
\hline Cesárea & 44 & 26 & 2,27 & 0,007 \\
\hline Vaginal o fórceps & 56 & 75 & $(1,20-4,30)$ & \\
\hline \multicolumn{5}{|l|}{ Tiempo proyección LME } \\
\hline $0-6$ meses & 52 & 41 & 1,61 & 0,098 \\
\hline 7 o más meses & 44 & 56 & $(0,88-2,97)$ & \\
\hline \multicolumn{5}{|l|}{ Apoyo paterno } \\
\hline No & 8 & 7 & 1,17 & 0,795 \\
\hline Sí & 92 & 94 & $(0,37-3,76)$ & \\
\hline Edad materna (años) & 29,9 & 29,4 & & $0,529^{*}$ \\
\hline Peso de nacimiento (g) & 3.436 & 3.326 & & $0,041^{*}$ \\
\hline
\end{tabular}

OR, odds ratio; *Valor p t-student.

tituto lácteo, el resto dos o más sustitutos (el rango fue de 1 hasta 12 suplementos lácteos). El suplemento principalmente usado fue suero glucosado $(78 \%)$, el $21 \%$ restante recibió fórmula láctea. Los recién nacidos suplementados presentaron significativamente mayor promedio de peso de nacimiento $(+110 \mathrm{~g} ; \mathrm{p}<0,041)$, una mayor chance de haber nacido por cesárea $(\mathrm{OR}=2,27 ; \mathrm{p}=0,007)$ y una mayor chance de que sus madres tengan 13 o más años de estudios $(\mathrm{OR}=1,94 ; \mathrm{p}=0,041)$. El modelo de regresión log binomial construido para determinar el riesgo ajustado de cese de LME al mes de vida incluyó la principal variable de exposición (suplementación) y las tres covariables anteriormente mencionadas, siendo el
RR ajustado $=1,54$ (IC95\% 1,01-2,35).

Por último, el modelo de regresión logística multivariado construido en base a las covariables asociadas en forma significativa a la principal variable de exposición demostró que la única variable (ajustada) asociada en forma significativa a la suplementación del recién nacido en la Maternidad fue la vía de parto (OR ajustado $=2,21$; IC95\% 1,20-4,05), siendo 2,2 veces más frecuente la suplementación en aquellos recién nacidos vía cesárea. Con respecto a las causas de suplementación en puerperio, el $31 \%$ fue por percepción de recién nacido demandante, $13 \%$ por dolor al amamantar por grietas y $11 \%$ por escaso calostro (figura 2). 


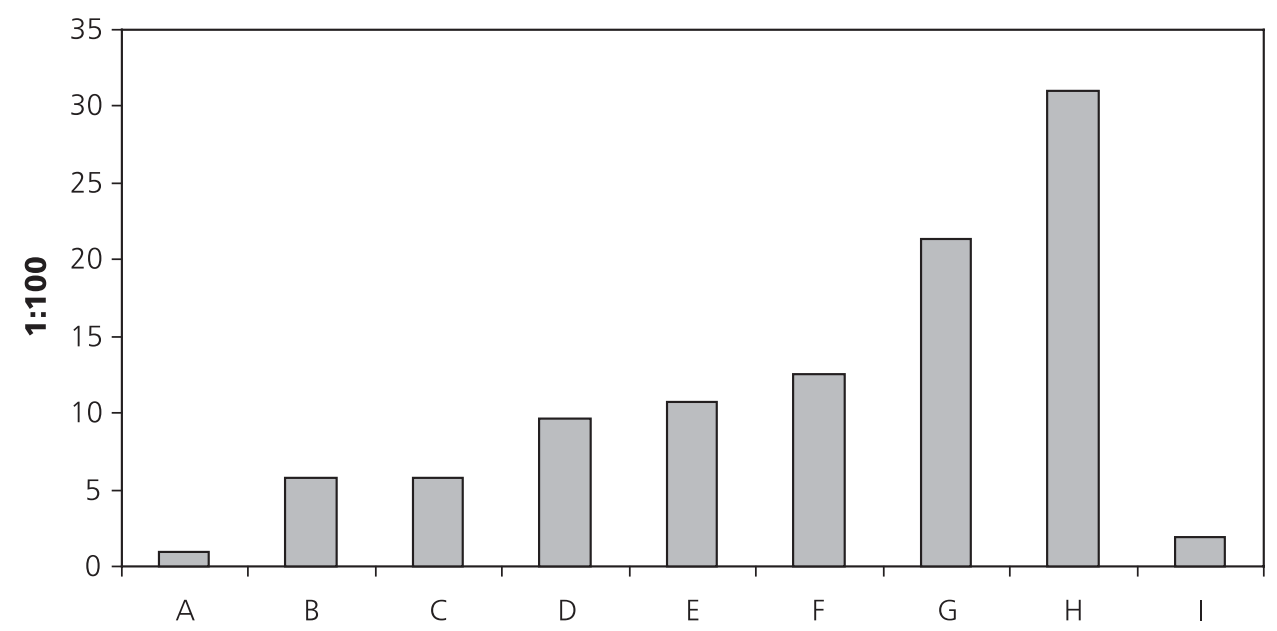

Figura 2. Causas de suplementación en puerperio ( $n=103$ binomios madre-hijo). A: Problemas de acople madre-hijo. B: Hipoglicemia neonatal $(<45 \mathrm{mg} / \mathrm{dL})$. C: Baja de peso mayor al 10\%. D: Madre imposibilitada por procedimiento. E: Escaso calostro. F: Grietas del pezón. G: No especificado. H: Recién nacido demandante. I: Otros.

\section{Discusión}

El presente estudio demuestra una asociación estadísticamente significativa entre la introducción de SLM u otros líquidos durante la estadía del recién nacido en la Maternidad y el cese de LME al mes de vida, tanto en forma cruda como ajustada. En la literatura, escasos trabajos han evaluado los efectos que esta práctica pudiese tener sobre la mantención de la $\mathrm{LME}^{8,9,11,12,22}$. Si bien existen condiciones médicas que exigen la suplementación transitoria tales como hipoglicemia neonatal, baja de peso extrema y procedimientos que imposibiliten temporalmente a la madre amaman$\operatorname{tar}^{13,23}$, éstas son más bien infrecuentes. Por el contrario, las principales razones por las cuales se utilizan SLM u otros líquidos son angustia materna ante un niño demandante, dificultades en el acoplamiento entre madre e hijo y formación de grietas, causas que también se ha encontrado en otros trabajos $5,7-9,13,16,18,22,23$, siendo éstas en su mayoría manejables por el equipo de salud ${ }^{14,18,24}$. En este trabajo, la percepción de niño demandante y dificultades en la técnica de amamantamiento explicaron más del $40 \%$ de las causas de suplementación, siendo mandatoria la guía, acompañamiento y prevención de este tipo de problemas por el equipo de sa$\operatorname{lud}^{6,7,14,20,24,25}$. Por su parte, la hipoglicemia y baja de peso significativa podrían ser disminuidas con el inicio de la lactancia materna durante la primera hora de vida ${ }^{19}$, en especial si se considera que el riesgo de baja de peso significativo es 7,1 veces mayor cuando se retrasa el inicio de la lactancia materna ${ }^{8,26}$.

Entre los factores asociados a la práctica de suplementación, tanto en forma univariada como multivariada los recién nacidos suplementados presentaron significativamente mayor frecuencia de nacimientos por cesárea, situación que podría explicarse por el hecho de que luego de realizarse esta cirugía se requiere más tiempo para iniciar la lactogénesis l,26,27; $^{8}$ de igual forma, las madres refieren más dolor post procedimiento, siendo común el uso de sustitutos de leche materna u otros líquidos ${ }^{8,22,26}$. Desde un punto de vista de salud pública, esta situación no deja de ser preocupante si se considera que la tasa de cesáreas de Chile es la más alta de los países latinoamericanos ${ }^{28-30}$, situación que confiere una mayor probabilidad de utilizar SLM u otros líquidos precozmente y de cese de LME al mes de vida ${ }^{31}$.

Es una política de salud al interior del Hospital Clínico de la Universidad Católica fomentar la práctica de la lactancia materna antes de la primera hora de vida del recién nacido y durante la estadía de las madres en la Maternidad, realizándose educación diaria y charlas 
grupales sobre este tema. Se cuenta además con un equipo de Clínica de Lactancia, el cual mantiene el apoyo y educación de las madres posterior al alta. Los resultados del presente estudio nos motivan a plantear la necesidad de optimizar la capacitación del equipo de salud, comenzando por la identificación de madres de mayor riesgo de introducción de SLM, así como disponer de pautas claras de indicación de SLM en cada Maternidad, medidas que esperamos permitan realizar un manejo adecuado de las dificultades presentes en el inicio de la $\mathrm{LME}^{19}$.

Desde un punto de vista metodológico, si bien una de las principales fortalezas del presente estudio es su carácter prospectivo, su diseño no está ajeno a la presencia de limitaciones. Su carácter observacional lo hace más vulnerable a la presencia de sesgo, debido a que ambas cohortes (suplementados vs nosuplementados) no difieren únicamente en la principal variable de exposición, existiendo un conjunto de variables confundentes por las cuales es necesario ajustar la asociación en estudio. Por tal razón, limitamos los hallazgos del presente estudio tan sólo a una asociación entre variables, sin plantear una relación de tipo causa-efecto entre ambas. Una segunda limitante del presente estudio tiene relación con su validez externa, considerando que el universo al cual pueden generalizarse estos resultados es relativamente acotado (recién nacidos de término, sanos, nacidos en una Maternidad con ciertas características particulares). No obstante ello, esperamos que esta experiencia sea un estímulo para la investigación en este tema por parte de otros equipos, cuyos resultados presenten mayores niveles de generalización, todo ello con el fin de promover las mejores prácticas de salud en la población.

\section{Referencias}

1.- American Academy of Pediatrics: Work Group on Breastfeeding. Breastfeeding and the use of human milk. Pediatrics 1997; 100:1035-9.

2.- Organización Mundial de la Salud: La alimentación del lactante y del niño pequeño. Disponible en: http:// whqlibdoc.who.int/publications/2010/9789275330944_ spa.pdf. Ultima visita: 22/04/10.
3.- Philipp B, Merewood A: The baby-friendly way: the best breastfeeding start. Pediatr Clin North Am 2004; 51: 761-83.

4.- Atalah E: Situación Actual de la Lactancia en Chile. Disponible en http://www.medwave.cl/cursos/pediatraynutricion06/1/1.act. Ultima visita 22/04/10.

5.- Gianni M, Vegni C, Ferraris $G$, et al: Usefulness Of An Early Breastfeeding Assessment Score to Predict Exclusive Breastfeeding Failure. Pediatr Res 2004; 56: 480.

6.- American Academy of Pediatrics: Policy Statement. Organizational Principles to Guide and Define the Child Health Care System and/or Improve the Health of All Children. Section on Breastfeeding. Breastfeeding and the Use of Human Milk. Pediatrics 2005; 115: 496-506.

7.- Taveras E, Capra A, Braveman P, Jensvold N, Escobar $G$, Lieu T: Clinician Support and Psychosocial Risk Factors Associated With Breastfeeding Discontinuation. Pediatrics 2003; 112: 108-15.

8.- Dewey K, Nommsen-Rivers L, Heinig M, Cohen R: Risk Factors for Suboptimal Infant Breastfeeding Behavior, Delayed Onset of Lactation, and Excess Neonatal Weight Loss. Pediatrics 2003; 112: 607-19.

9.- Cronenwett L, Stukel T, Kearney M, et al: Single Daily Bottle Use in the Early Weeks Postpartum and BreastFeeding Outcomes. Pediatrics 1992; 90: 760-6.

10.- Barría M, Orozco B, Gatica M, Mackenney J, Valverde $C$, Drago M, Valencia C: Introducción Precoz de Fórmulas Lácteas en la Alimentación del Niño. Rev Chil Pediatr 1990; 61: 218-22.

11.- Martin-Calama J, Buftuel J, Valero M, et al: The Effect of Feeding Glucose Water to Breastfeeding Newborns on Weight, Body Temperature, Blood Glucose, and Breastfeeding Duration. J Hum Lact 1997; 13: 209-13.

12.- Hill P, Humenick S, Brennan M, Woolley D: Does Early Supplementation Affect Long-Term Breastfeeding?. Clin Pediatr (Phila) 1997; 36: 345-50.

13.- Neifert $M$ : Prevention of Breastfeeding Tragedies. Pediatr Clin North Am 2001; 48: 273-93.

14.- Kramer MS, Chalmers B, Donet E: Promotion of Breastfeeding Intervention Trial (PROBIT) A Randomized Trial in the Republic of Belarus. JAMA 2001; 285: 413-20.

15.- Feinstein J, Berkelhamer J, Gruszka M, Wong C, Carey $A$ : Factors related to early termination of breast-feeding in an urban population. Pediatrics 1986; 78: 210-5.

16.- Hill P, Aldag J, Chatterton R, Zinaman M: Primary and Secondary Mediators Influence on Milk Output in Lactating Mothers of Preterm and Term Infants. J Hum Lact 2005; 21: 138-50. 
17.- Szajewska H. Horvath A, Berthold K, Mercin K: Effects of brief exposure to water, breast-milk substitutes, or other liquids on the success and duration of breastfeeding: A systematic review. Acta Paediatr 2006; 95: 145 52.

18.- Wagner C, Wagner M: The Breast or the Bottle? Determinants of Infant feeding Behaviors. Clin Perinatol. 1999; 26: 505-24.

19.- Berens P: Prenatal, Intrapartum, and Postpartum Support of the Lactating Mother. Pediatr Clin North Am 2001; 48: 365-75.

20.- Lawrence P: Breast Milk. Best Source of Nutrition for Term and Preterm Infants. Pediatr Clin North Am. 1994; 41: 925-40.

21.- Hill P, Aldag J, Chatterton R, Zinaman M: Primary and Secondary Mediators' Influence on Milk Output in Lactating Mothers of Preterm and Term Infants. J Hum Lact 2005; 21: 138-50.

22.- Gray-Donald K, Kramer M, Munday S: Effect of Formula Supplementation in the Hospital on the Duration of Breast-Feeding: A Controlled Clinical Trial. Pediatrics 1985; 75: 514-8.

23.- Howard C, Howard F, Lanphear B, et al: Randomized Clinical Trial of Pacifier Use and Bottle-Feeding or Cupfeeding and Their Effect on Breastfeeding. Pediatrics 2003; 111: 511-8.

24.- Taveras E, Li R, Grummer-Strawn L, et al: Opinions and Practices of Clinicians Associated With Continuation of Exclusive Breastfeeding. Pediatrics 2004; 113: e283-e290.

25.- Kuan L, Britto M, Decolongon J, Schoettker P, Athertton $H$, Kotagal U: Health System Factors Contributing to Breastfeeding Success. Pediatrics 1999; 104: 1-7.

26.- Sievers E, Haase S, Oldigs H, Schaub J: The Impact of Peripartum Factors on the Onset and Duration of Lactation. Biol Neonato 2003; 83: 246-52.

27.- Neville M, Morton J, Umemura S: Lactogenesis. Pediatr Clin North Am 2001; 48:35-50.

28.- Belizán J, Althabe F, Barros F, Alexander S: Rates and implications of caesarian sections in Latin America: ecological study. BMJ 1999; 319: 1397-400.

29.- Latorre R, Carrillo J, Yamamoto M, et al: Gobierno del Parto en el Hospital Padre Hurtado: un Modelo para contener la Tasa de Cesárea y Prevenir la Encefalopatía Hipóxico-Isquémica. Rev Chil Obstet Ginecol 2006; 71: 196-200.

30.- Cabrera J, Cruz G, Cabrera C, et al: Características del Peso, Edad Gestaccional y Tipo de parto del RN en el Sistema Público y Privado. Rev Chil Obstet Ginecol 2006; 71: 92-7.

31.- Hauck YL, Fenwick J, Dhaliwal SS, Butt J: A Western Australian Survey of Breastfeeding Initiation, Prevalence and Early Cessation Patterns. Matern Child Health J 2010 (Epub ahead of print). 